\title{
Guided mode resonance flat-top bandpass filter for terahertz telecom applications
}

\author{
A. Ferraro, ${ }^{1, *}$ (i) A. A. Tanga, ${ }^{2}$ D. C. Zografopoulos, ${ }^{1}$ (i) G. C. Messina, ${ }^{2,3}$ M. Ortolani, ${ }^{2}$ (i) \\ AND R. BeCCHERELLI ${ }^{1}$ (1) \\ ${ }^{1}$ Consiglio Nazionale delle Ricerche, Istituto per la Microelettronica e Microsistemi (CNR-IMM), Roma 00133, Italy \\ ${ }^{2}$ Department of Physics, Sapienza University of Rome, Rome 00185, Italy \\ ${ }^{3}$ Consiglio Nazionale delle Ricerche, Istituto dei Sistemi Complessi (CNR-ISC), Rome 00185, Italy \\ ${ }^{*}$ Corresponding author: antonio.ferraro@artov.imm.cnr.it
}

Received 15 July 2019; accepted 30 July 2019; posted 1 August 2019 (Doc. ID 372588); published 22 August 2019

In this work, we design and experimentally demonstrate a novel terahertz (THz) filter exhibiting a flattened spectral response in the atmospheric transmission window around the central frequency of $300 \mathrm{GHz}$. The innovative concept behind this filter is the coupling of Fabry-Perot and guided mode resonances. The latter arise from a two-dimensional patch array patterned on an aluminum layer deposited on a low loss cyclo-olefin polymer. The filter experimental performance shows high transmittance in the flat-top band, with less than $3 \mathrm{~dB}$ losses, and high out-of-band rejection, as theoretically expected. This kind of component provides a cost-effective, functional solution for narrowband filtering in emerging $\mathrm{THz}$ devices and systems with possible applications in wireless telecommunications. () 2019 Optical Society of America

https://doi.org/10.1364/OL.44.004239

Recent years have seen a progressive filling of the so-called terahertz $(\mathrm{THz})$ gap thanks to the developing of novel quasi-optical devices to control and manipulate $\mathrm{THz}$ waves, such as polarizers, waveplates, lenses, modulators, filters, and so on [1-6]. These innovative components have boosted the application of $\mathrm{THz}$ waves with improved performance in several fields like agriculture, pharmaceutics, biology, and security [7]. Among them, particular attention has been addressed to the field of telecommunications, both by the microwave and optics scientific communities [8]. In fact, the huge demand for data traffic keeps pushing the carrier frequency towards upper limits in order to increase the available bandwidth and, hence, the amount of data that can be transmitted. Currently, 5G networks use millimeter-wave frequencies, with frequency bands up to $29.5 \mathrm{GHz}$ already licensed in the European Union, the USA, and Japan. However, it is expected that the trend will continue in the future, encompassing millimeter waves possibly up to $86 \mathrm{GHz}$. Then, further growth is envisaged in the $\mathrm{D}$ band and in the $\mathrm{THz}$ range. A promising window for telecom applications falls in the $200-320 \mathrm{GHz}$ band, which enables the transmission of tens of gigabits (Gbit/s) [9]. To this aim, high-quality bandpass filters are indispensable in order to separate transmission/reception channels and to avoid crosstalking among them. An ideal approach is represented by the so-called flat-top bandpass filters, characterized by a rectangular transfer function. In practice, the ideal response is approximated by a high and flat transmittance value within the pass-band rapidly decaying outside the range. In the visible and infrared (VIS/IR) frequency range, a common method for the realization of flattop filters is to alternate tens/hundreds of thin layer coatings of materials with different dielectric constants [10]. However, this approach is unfeasible at $\mathrm{THz}$ frequencies due to the difficulty to precisely alternate such a high number of layers and to the scarcity of materials with the required dielectric constant values and low absorption loss.

A novel approach is represented by the cascading of two or more guided mode resonance (GMR) filters, thus realizing a Fabry-Perot (FP) cavity, where the FP and GMR resonances are coupled to produce a flat-top response [11-13]. This technique was recently proposed in the VIS/IR spectrum, although it has not yet been experimentally validated. A GMR occurs at a frequency where the first-order diffracted wave of a grating is coupled to a mode guided in an adjacent dielectric slab waveguide. This concept has been known in optics since the pioneering work of Magnusson's group in 1992 [14]. However, it has been applied to $\mathrm{THz}$ frequencies only recently $[15,16]$. In a previous work, we exploited the concept of GMR by fabricating a series of $\mathrm{THz}$ filters operating in the $625-725 \mathrm{GHz}$ and 780 $910 \mathrm{GHz}$ telecom windows. The filters showed very high transmission value ( $T \sim 86 \%)$, high-quality factors $(Q \sim 70)$, and high out-of-band rejection [17]. GMRs are also observed as secondary effects into classic frequency selective surface (FSS) aluminum filters patterned on a low loss substrate $[18,19]$.

In this work, we demonstrate the proof-of-principle of a $\mathrm{THz}$ flat-top filter operating in the high-frequency edge of the 200$320 \mathrm{GHz}$ telecom window obtained by cascading two identical GMR filters at an appropriate distance, hereafter referred to as a FP-GMR filter. The proposed filter enables the allocation of dedicated spectral channels for different application/operators or for signal transmission and reception. To the best of our knowledge, this is the first time that a flat-top filter based 
on cascading two GMR filters was fabricated and measured at $\mathrm{THz}$ frequencies. After a detailed numerical analysis, we fabricated the filter on a substrate of Zeonor, a low loss cyclo-olefin polymer, coated with $200 \mathrm{~nm}$ of aluminum. The periodic twodimensional (2D) patch arrays were defined by means of standard UV photo-lithography. Then, the filter was experimentally characterized, thus validating the proof-of-concept.

Figure 1(a) shows a schematic three-dimensional (3D) model (left) of the proposed FP-GMR filter. Two identical polarization-insensitive GMR filters are placed at a finite distance $d$, thus enabling the coupling of the GMR resonance to those of the resulting FP cavity. A photograph of one of the fabricated single GMR filters is reported in Fig. 1(b). The thickness $t$ of the polymer substrate, the grating period along the two grating axes $\left(p_{x}=p_{y}\right)$, and the width $w$ of the aluminum patch define the spectral response of the single GMR filter. Thanks to the C4 symmetry, the proposed filter is polarization insensitive [17].

Figure 2 shows a detailed numerical analysis performed by using the rigorous coupled wave analysis (RCWA) [20] for a filter with period, patch width, and substrate thickness of $765 \mu \mathrm{m}, 689 \mu \mathrm{m}$, and $385 \mu \mathrm{m}$, respectively. The resulting resonant frequency $f_{0}$ is at $300 \mathrm{GHz}$. We modeled aluminum as a Drude medium and Zeonor as a dielectric with a refractive index equal to $n_{z}=1.525-j 0.0013$ [17]. Figure 2(a) studies the spectral response of the single GMR filter for a perpendicularly impinging plane wave. Thanks to the low loss of the polymer substrate, the power absorption is very low at the resonance

(a)

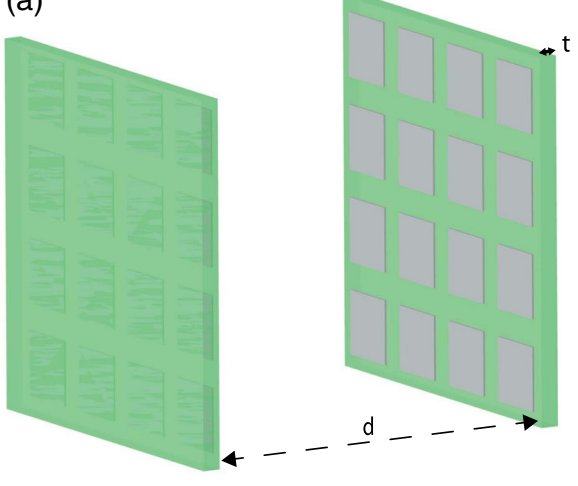

(b)

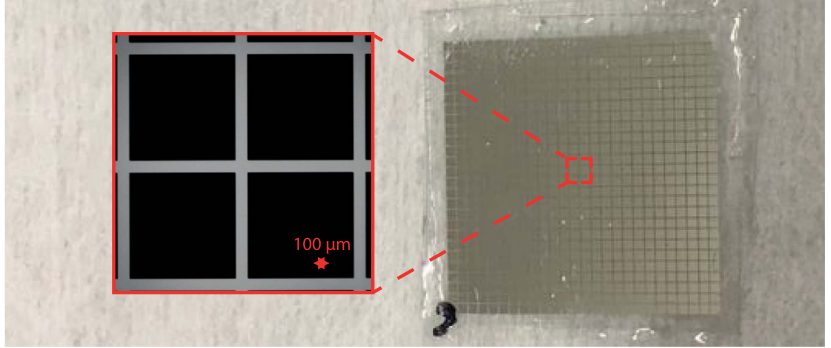

Fig. 1. (a) (Left): schematic 3D diagram of the Fabry-Perot guided mode resonance (FP-GMR) filter composed of two identical GMR filters separated by a distance $d$. (Right): unit cell, where $p_{x}$ and $p_{y}$ is the lattice period along the $x$ and $y$ axis, respectively, and $w$ is the patch width. (b) Microscope picture taken in transmission mode and photograph of the fabricated single GMR.
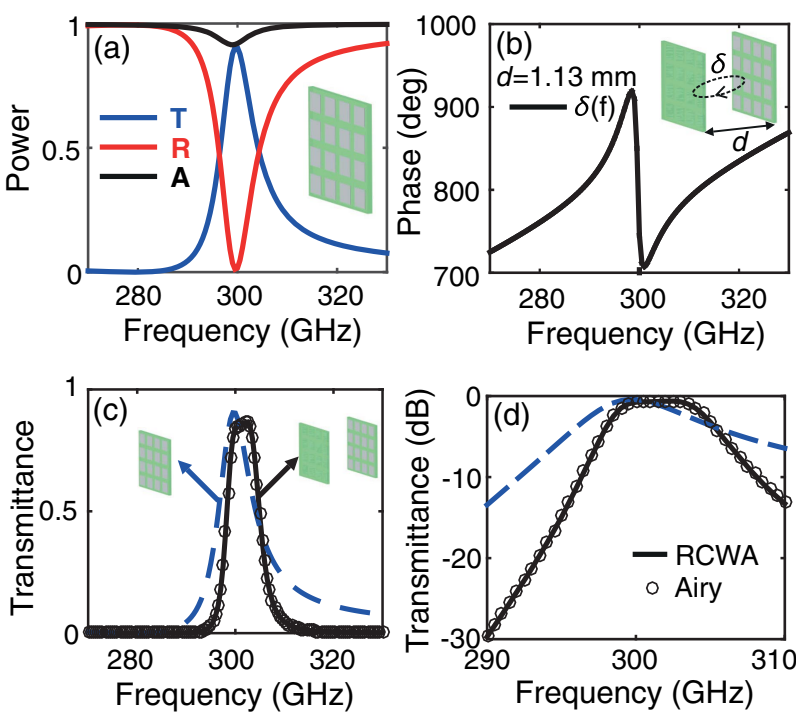

Fig. 2. Numerically calculated (a) transmittance (blue line), reflectance (red line), and absorption (black line) for a single GMR filter. (b) Phase difference for two identical GMR filters with separation of $1.13 \mathrm{~mm}$. (c) Comparison of transmittance between a single GMR (blue line) and FP-GMR at $1.13 \mathrm{~mm}$ (black line). (d) Zoom of the comparison expressed in decibel $(\mathrm{dB})$.

frequency and negligible elsewhere, while the peak transmittance reaches almost $90 \%$ at $f_{0}$.

The response of the FP-GMR filter is described by the Airy function:

$$
T_{e}=\frac{T^{2}}{1-2 R \cos \delta(\lambda)+R^{2}},
$$

where $T$ and $R$ are the single GMR filter transmittance and reflectance, respectively, and $\delta(f)$ is the round-trip phase, as shown in the inset of Fig. 2(b), given by

$$
\delta(f)=\left(\frac{4 \pi d f}{c}\right)-2 \varphi_{R}(f),
$$

where $c$ is the speed of light in vacuum, and $\varphi_{R}$ is the phase of the reflected wave of the single GMR [11]. By controlling the separation distance between the two GMR filters, it is possible to tune the response of the FP-GMR filter. In particular, a value of $d=1.13 \mathrm{~mm}$ corresponds to the round-trip phase profile shown in Fig. 2(b), achieving a flat-top transmission band at around $300 \mathrm{GHz}$. This coupling effect is demonstrated in Figs. 2(c) and 2(d), which report a direct comparison in the linear and logarithmic scales, respectively, of the FP-GMR filter transmittance versus that of the single GMR filter.

A 5-GHz-wide flat-top band is observed for the FP-GMR filter, showing steeper and higher out-of-band rejection values with respect to the single GMR filter.

The FP-GMR filter transmittance was calculated by using both the Airy function approximation and a full RCWA simulation. As expected, the values obtained by the two approaches are in excellent agreement, due to the lack of diffractive contributions to the investigated frequency window. Therefore, once a single GMR filter is characterized, the Airy function 

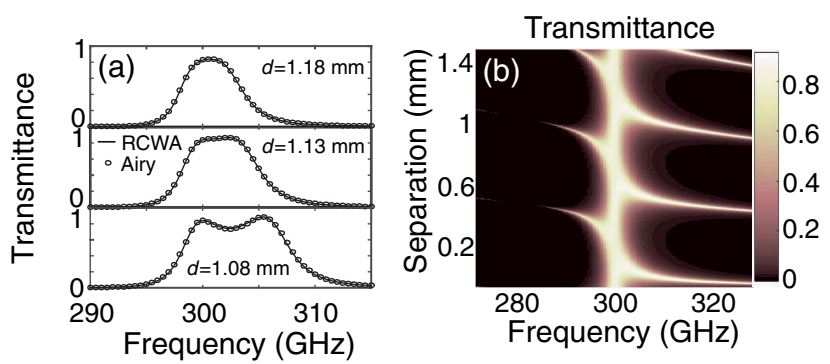

Fig. 3. Numerically calculated transmittance of the FP-GMR filter for (a) three separation distance values and (b) as a function of separation value for $d$ ranging from 0.1 to $1.5 \mathrm{~mm}$.

can be directly employed for the fast simulation/optimization of the coupled system.

Figure 3 reports the dependence of the transmittance of the FP-GMR filter on the separation distance. In particular, Fig. 3(a) shows the filter transmittance for the optimal value of $1.13 \mathrm{~mm}$ and offsets of $\pm 50 \mu \mathrm{m}$. The separation value influences the shape of the flat-top band without modifying the transmittance value at the central frequency. Since the filter transmittance depends on the round-trip phase, a periodic behavior should be expected. This periodicity is clearly visible in Fig. 3(b), which reports the transmittance calculated in the frequency window of interest for separation values from 0.1 to $1.5 \mathrm{~mm}$. Such behavior provides additional degrees of freedom in the fabrication process, since the optimal $d$ value can be chosen from a set of discrete values.

After the numerical design, the single GMR filters were fabricated on a $385-\mu \mathrm{m}$-thick Zeonor substrate with a square surface of $2.54 \mathrm{~cm} \times 2.54 \mathrm{~cm}$ using standard UV photolithography. A 1.3- $\mu \mathrm{m}$-thick positive photoresist (S1813 from Shipley) was spin coated and baked on top of a previously evaporated $200 \mathrm{~nm}$ aluminum layer. Then, the photoresist was exposed to UV light, developed with MF319, and rinsed with deionized water. The exposed aluminum (area not protected by the resist) was wet-etched in a solution of $\mathrm{H}_{3} \mathrm{PO}_{4}: \mathrm{H}_{2} \mathrm{O}: \mathrm{CH}_{3} \mathrm{COOH}: \mathrm{HNO}_{3}=16: 2: 1: 1$. Finally, the sample was cleaned with acetone and isopropyl alcohol (IPA) in order to remove the residual photoresist and dried with nitrogen flow.

The microscope picture in the inset of Fig. 1(b) shows a portion of the fabricated GMR filter having patches with a width of $689 \mu \mathrm{m}$ and a final grating period of $765 \mu \mathrm{m}$.

The filters were measured by means of a home-built setup, shown in Fig. 4(a), with high accuracy in the placement of each GMR filter. The incident angle and the parallelism of the two filters were carefully checked by using an external red laser aligned with the $\mathrm{THz}$ wave. A THz transmission spectrometer was constructed so as to obtain a perfectly collimated beam for all frequencies of interest. To this aim, we employed a voltagecontrolled oscillator [VCO, yttrium iron garnet (YIG)-type, from Microlambda] connected to an amplifier-multiplier chain based on Schottky diode multipliers [analyzer module controller (AMC) from Virginia Diodes Tx-175]. The VCO was tuned stepwise with a Keithley precision voltage source to generate monochromatic waves at frequencies between 290 and $320 \mathrm{GHz}$ with steps of $1 \mathrm{GHz}$. A WR2.8 diagonal horn antenna after the last multiplier acts as a point source emitting
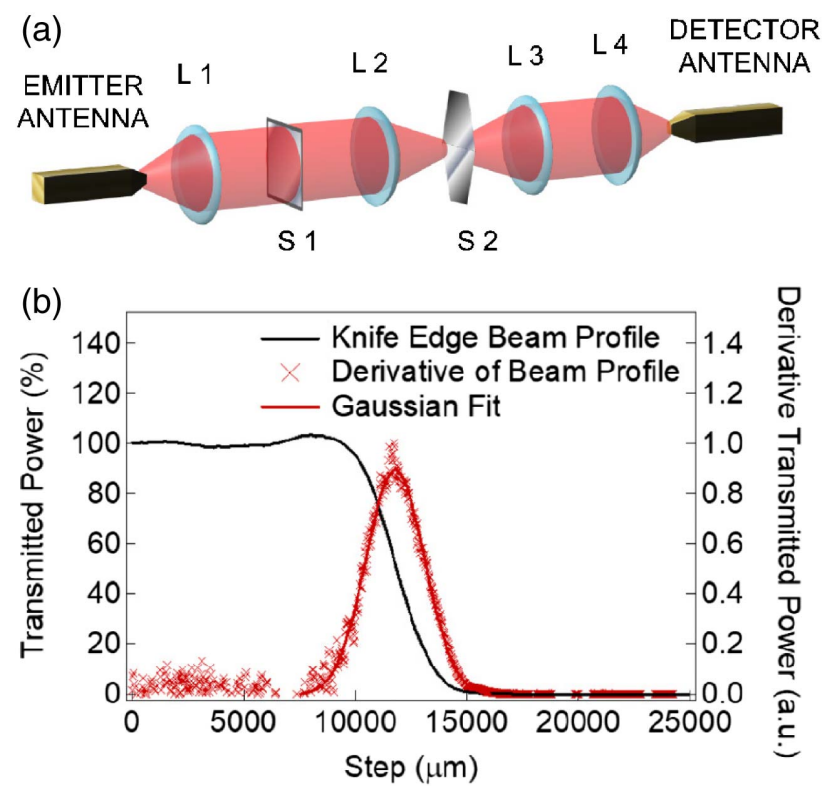

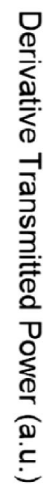

Fig. 4. (a) Schematic diagram of the $\mathrm{THz}$ transmission setup: L1, $\mathrm{L} 2, \mathrm{~L} 3$, and $\mathrm{L} 4$ are $\mathrm{THz}$ lenses, $\mathrm{S} 1$ is the filter, and $\mathrm{S} 2$ is the razor blade. (b) Knife-edge experimental results.

a Gaussian beam in free space with $6^{\circ}$ divergence (half width at half-maximum), linearly polarized along the vertical direction with respect to the optical table. In fact, the horn antenna transforms the rectangular waveguide mode TE10 into a pure transverse electromagnetic (TEM00) mode [21], which can be manipulated optically [22], in this case to obtain a perfectly collimated beam. To do so, we precisely positioned a plano-convex polymethylpentene (TPX) lens $(D=50 \mathrm{~mm}$, $f=75 \mathrm{~mm}$ ) with its focus coinciding with the virtual focus of the WR2.8 horn antenna [23], indicated as L1 in Fig. 4(a). The collimated beam diameter calculated by geometrical optics is $16 \mathrm{~mm}$, i.e., smaller than the sample side of $25.4 \mathrm{~mm}$, but large enough to avoid diffraction broadening in the short collimated beam path of $40 \mathrm{~mm}$ between the lens and sample. The quality of beam collimation has been checked by positioning a second TPX lens (L2, $D=50 \mathrm{~mm}, f=100 \mathrm{~mm}$ ) after the sample position [S1 in Fig. 4(a)] and performing a knife-edge scan with a razor blade [S2 in Fig. 4(a)] to confirm diffractionlimited spot size. The result shown in Fig. 4(b) reveals a FWHM of $3.0 \mathrm{~mm}$ at $298 \mathrm{GHz}$, as expected [24]. As shown in Fig. 4(a), a Schottky diode zero-bias rectifier positioned in a focus of L4 and connected to a lock-in amplifier was finally employed to measure the transmitted power for each generated frequency (on-off amplitude modulation at $1 \mathrm{kHz}$ was applied to the AMC circuit) [22]. The Keithley voltage source and the lock-in amplifier were connected to a computer with custom software for automated acquisition of identical spectra for each sample and reference.

The experimentally measured transmittance of both the single GMR and coupled FP-GMR filter system are in good agreement with the simulations illustrated in Fig. 5. The experiments on the FP-GMR filter have shown a central frequency of $300 \mathrm{GHz}$ with a bandwidth of $19 \mathrm{GHz}$ at $-15 \mathrm{~dB}$, which is similar to the value of $16 \mathrm{GHz}$ obtained by numerical simulations. Moreover, it is evidenced that the 


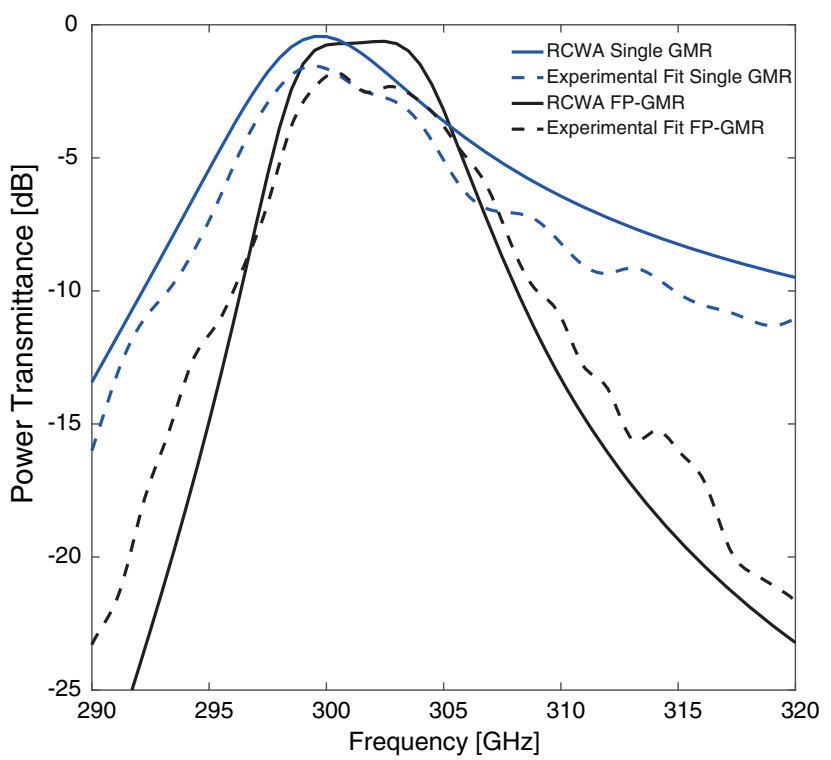

Fig. 5. Numerically and experimentally measured transmittance of single GMR (blue line) and FP-GMR flat-top filter (black line).

single GMR filter does not exhibit a flat-top response in the transmission band and shows much higher transmittance value out-of-band. The discrepancy between numerical and experimental results can be attributed to misalignment effects, resulting in a slight variation of the distance between the two single GMR filters.

To sum up, we have proposed a proof-of-principle $\mathrm{THz}$ filter operating at $300 \mathrm{GHz}$ showing flat-top response with less $1 \mathrm{~dB}$ in band ripple and rapid decay of the transmission band obtained by cascading two GMR filters. The filters were placed at distance of $1.13 \mathrm{~mm}$, thus creating a FP cavity. The experimental measurements are in very good agreement with numerical simulations, reporting a flat band of $5 \mathrm{GHz}$ and bandwidth of 19 at $-15 \mathrm{~dB}$. The flat-top properties of the proposed filter make it suitable for operation in $\mathrm{THz}$ wireless telecom applications, where it is necessary to separate each communication channel with high precision.

Funding. "European Network for High Performance Integrated Microwave Photonics" European Commission (COST Action CA16220).

\section{REFERENCES}

1. L. Wang, X.-W. Lin, W. Hu, G.-H. Shao, P. Chen, L.-J. Liang, B.-B. Jin, P.-H. Wu, H. Qian, Y.-N. Lu, X. Liang, Z.-G. Zheng, and Y.-Q. Lu, Light Sci. Appl. 4, e253 (2015).

2. W. D. Furlan, V. Ferrando, J. A. Monsoriu, P. Zagrajek, E. Czerwińska, and M. Szustakowski, Opt. Lett. 41, 1748 (2016).

3. G. Isić, G. Sinatkas, D. C. Zografopoulos, B. Vasić, A. Ferraro, R. Beccherelli, E. E. Kriezis, and M. Belić, IEEE J. Sel. Top. Quantum Electron. 25, 8500108 (2019).

4. R. Yan, S. Arezoomandan, B. Sensale-Rodriguez, and H. G. Xing, ACS Photon. 3, 315 (2016).

5. A. Ferraro, D. C. Zografopoulos, R. Caputo, and R. Beccherelli, IEEE Photon. Technol. Lett. 28, 2459 (2016).

6. M. D. Astorino, R. Fastampa, F. Frezza, L. Maiolo, M. Marrani, M. Missori, M. Muzi, N. Tedeschi, and A. Veroli, Sci. Rep. 8, 1985 (2018).

7. H.-J. Song and T. Nagatsuma, Handbook of Terahertz Technologies: Devices and Applications (CRC Press, 2015).

8. T. Nagatsuma, G. Ducournau, and C. C. Renaud, Nat. Photonics 10, 371 (2016).

9. E. Lacombe, C. Belem-Goncalves, C. Luxey, F. Gianesello, C. Durand, D. Gloria, and G. Ducournau, IEEE Microw. Wireless. Compon. Lett. 28, 362 (2018).

10. H. A. Macleod, Thin-Film Optical Filters, 4th ed., Series in Optics and Optoelectronics (CRC Press, 2010).

11. D. K. Jacob, S. C. Dunn, and M. G. Moharam, Appl. Opt. 41, 1241 (2002).

12. Y. H. Ko and R. Magnusson, Opt. Lett. 41, 4704 (2016).

13. K. Yamada, K. J. Lee, Y. H. Ko, J. Inoue, K. Kintaka, S. Ura, and R. Magnusson, Opt. Lett. 42, 4127 (2017).

14. R. Magnusson and S. S. Wang, Appl. Phys. Lett. 61, 1022 (1992).

15. S. Song, F. Sun, Q. Chen, and Y. Zhang, IEEE Trans. Terahertz Sci. Technol. 5, 131 (2015).

16. H. S. Bark and T.-I. Jeon, Opt. Express 26, 29353 (2018).

17. A. Ferraro, D. C. Zografopoulos, R. Caputo, and R. Beccherelli, Sci. Rep. 8, 17272 (2018).

18. A. Ferraro, D. C. Zografopoulos, R. Caputo, and R. Beccherelli, IEEE J. Sel. Top. Quantum Electron. 23, 8501308 (2017).

19. A. Ferraro, D. C. Zografopoulos, R. Caputo, and R. Beccherelli, Appl. Phys. Lett. 110, 141107 (2017).

20. J. P. Hugonin and P. Lalanne, Reticolo Software for Grating Analysis (2005).

21. A. Kazemipour, M. Hudlička, R. Dickhoff, M. Salhi, T. KleineOstmann, and T. Schrader, J. Infrared Millim. Terahertz Waves 35, 720 (2014).

22. C. Ciano, M. Flammini, V. Giliberti, P. Calvani, E. DelRe, F. Talarico, M. Torre, M. Missori, and M. Ortolani, IEEE Trans. Terahertz Sci. Technol. 8, 390 (2018).

23. A. Garufo, P. M. Sberna, G. Carluccio, J. R. Freeman, D. R. Bacon, L. Li, J. Bueno, J. Baselmans, E. Linfield, A. G. Davies, N. Llombart, and A. Neto, IEEE Trans. Terahertz Sci. Technol. 9, 221 (2019).

24. S. Tofani, D. C. Zografopoulos, M. Missori, R. Fastampa, and R. Beccherelli, J. Opt. Soc. Am. B 36, D88 (2019). 